\title{
Vertically Integrated Nanowire Field Effect Transistors
}

Josh Goldberger, Allon I. Hochbaum, Rong Fan, Peidong Yang

Department of Chemistry, University of California, Berkeley, California 94720 and

Materials Science Division, Lawrence Berkeley National Laboratory

\section{Supplemental information}

\section{Non-lithographic Positional control of Silicon nanowires}
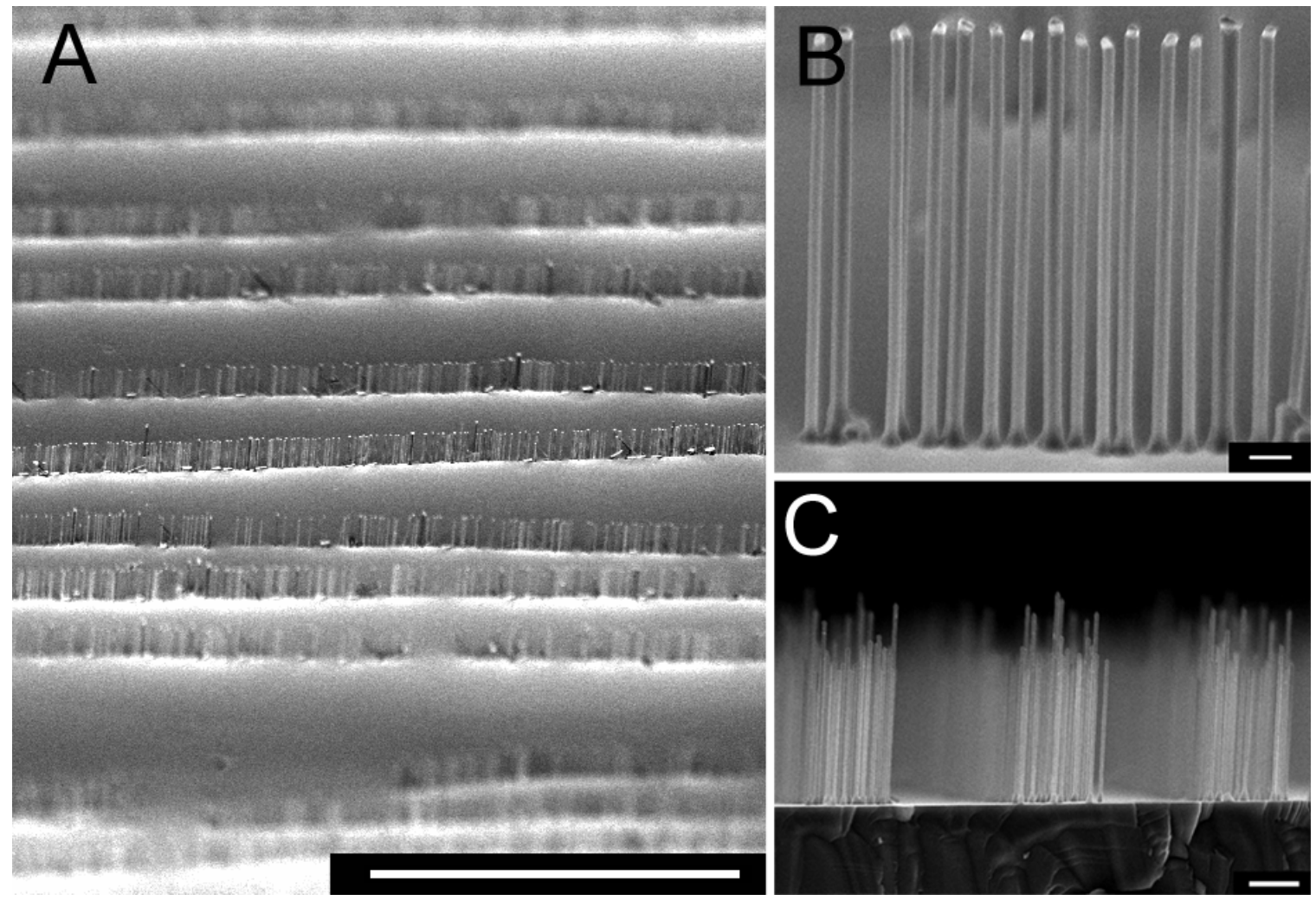

Fig. S1. Patterning of Silicon nanowires. (A) Overview and (B) zoomed in image of patterned lines of vertical Si nanowires grown from lines of single nanoparticle catalysts deposited onto a Silicon substrate via a Langmuir-Blodgett technique. ${ }^{1}$ (C) A crosssection SEM image of nanowires that were positionally aligned into lines via PDMS patterning of Au colloids. ${ }^{2}$ The scale bar in images (A), (B), and (C) correspond to 100 $\mu \mathrm{m}, 1 \mu \mathrm{m}$, and $1 \mu \mathrm{m}$, respectively. 


\section{VINFET fabrication procedure}

Si VINFETs are fabricated according to the following general processing procedure. After nanowire growth (Fig. S2a), the nanoparticle catalysts were etched away using aqua regia etching followed by a standard water and isopropanol rinse, and then $7 \mathrm{~min} \mathrm{O}_{2}$ plasma clean at $300 \mathrm{~W}$. Nanowires having diameters greater than $40 \mathrm{~nm}$ and aspect ratios less then 40 are mechanically resilient enough to remain vertical after the standard pregate oxide thermal oxidation cleaning procedure despite solvent surface tension forces (smaller diameters and larger aspect ratios were not measured). The substrates were oxidized at $850{ }^{\circ} \mathrm{C}$ for 4-32 hours to achieve the desired gate $\mathrm{SiO}_{2}$ dielectric thickness (Fig. S2b). Cr gate metal was sputtered to achieve a conformal 50-100 nm coating. (Fig. S2c). Alternatively, LPCVD techniques can be used to deposit the desired the gate materials (i.e. poly-Si, metal silicides) for threshold voltage tuning.

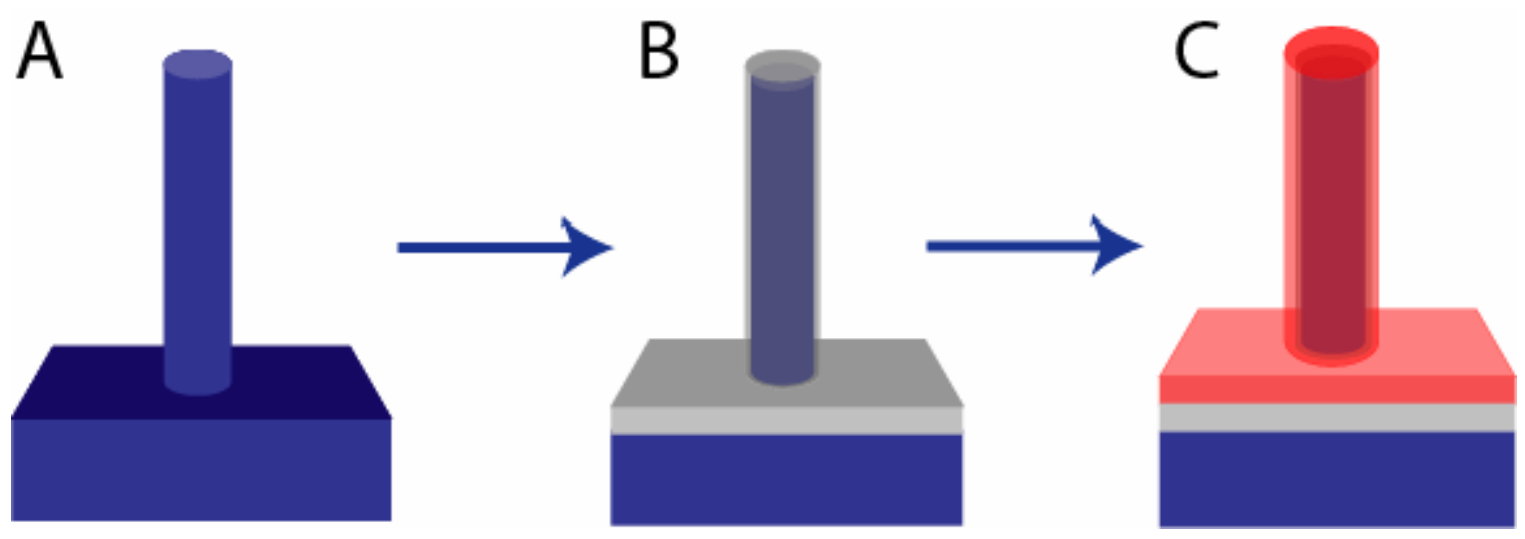

Fig. S2. Si VINFET fabrication. (A) Si nanowires are grown vertically from a Si (111) substrate. (B) Thermal oxidation of the Si nanowire is used to form the gate dielectric. (C) The $\mathrm{Cr}$ gate material is then sputtered onto the nanowires to achieve a conformal coating. Blue corresponds to the $\mathrm{Si}$ substrate and the Si nanowire channel, grey corresponds to the $\mathrm{SiO}_{2}$ dielectric material, and red corresponds to the $\mathrm{Cr}$ gate metal.

$750-4000 \mathrm{~nm}$ of conformal low pressure chemical vapor deposition (LPCVD) $\mathrm{SiO}_{2}$ dielectric was then deposited onto the substrates (Fig. S3a,d). A gate pattern was then defined via standard photolithographic techniques. After developing of the 
photoresist gate pattern, etch windows were created by plasma etching the exposed $\mathrm{SiO}_{2}$ areas using a LAM Research Corporation AutoEtch Plasma Etch System. The exposed Cr was removed using Cr-7 Photomask etchant from Cyantek.

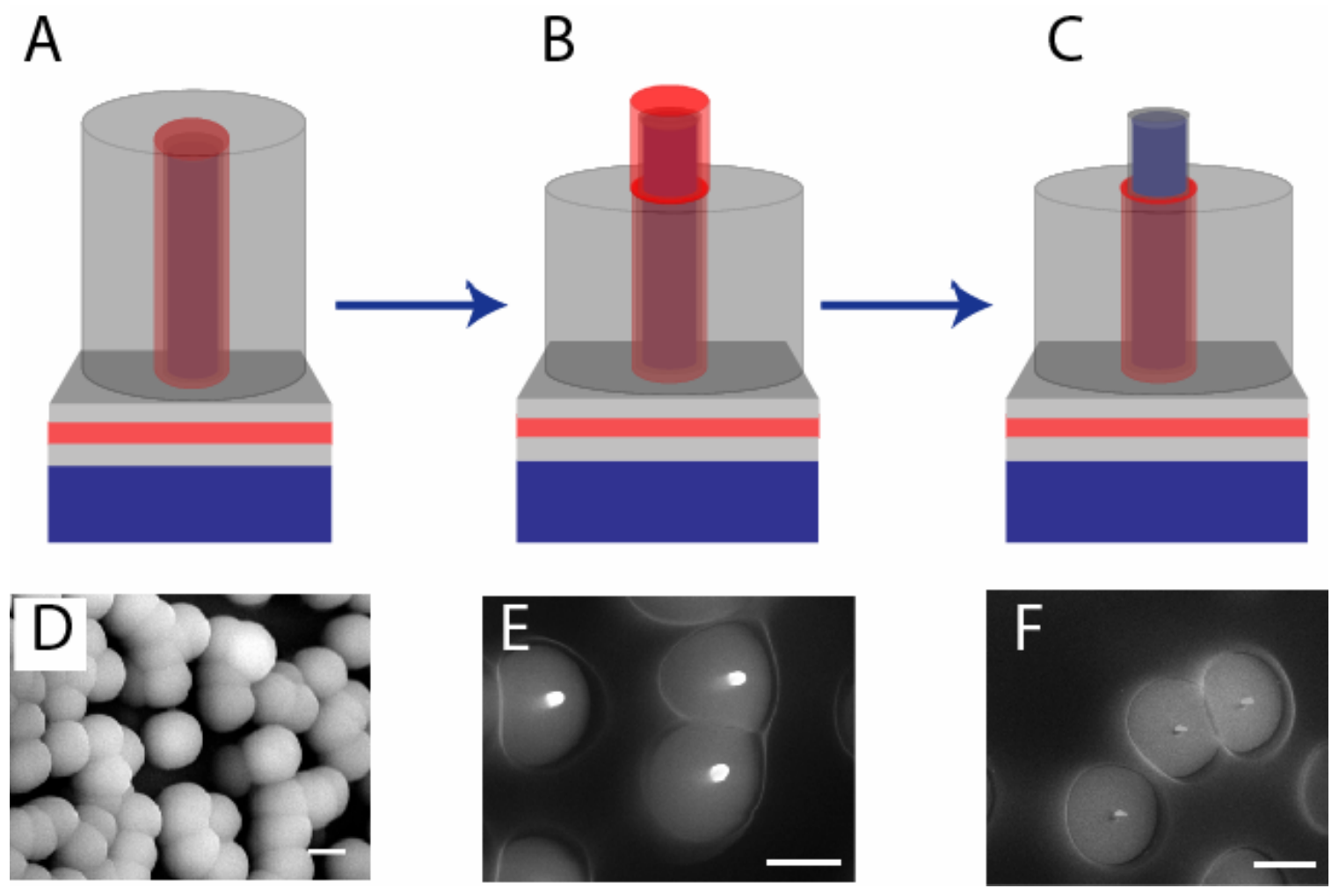

Fig. S3. Si VINFET fabrication. (A) Conformal LPCVD oxide is deposited around the nanowire. (B) The Cr-coated nanowire tips are exposed via chemomechanical polishing and plasma etching of the $\mathrm{SiO}_{2}$ dielectric. (C) The $\mathrm{Cr}$ gate material is etched-backed using a $\mathrm{Cr}$ photomask etchant. (D) An SEM image taken after the $\mathrm{SiO}_{2}$ deposition. (E) An SEM image showing the exposed Cr-coated tips. (F) an SEM image of the device after the $\mathrm{Cr}$ etch back procedure. All SEM scale bars correspond to $1 \mu \mathrm{m}$, and all images are obtained at a tilt angle of $30^{\circ} \mathrm{C}$. In the fabrication diagrams (A-C), blue corresponds to the $\mathrm{Si}$ substrate and the $\mathrm{Si}$ nanowire channel, grey corresponds to the $\mathrm{SiO}_{2}$ dielectric material, and red corresponds to the $\mathrm{Cr}$ gate metal.

At this point, the tips of the nanowires were still coated with the $\mathrm{Cr}$ gate metal. This material must be removed so as to prevent electrical shorting between the drain and gate electrodes. A combination of chemomechanical polishing and $\mathrm{SiO}_{2}$ plasma etching 
techniques was able to expose the nanowire tips (Fig. S3b,e). The Cr surrounding the tips of the nanowires was then removed using Cr-7 etchant (Fig. S3c,f).

A second $\sim 300-750 \mathrm{~nm}$ coating of $\mathrm{LPCVD} \mathrm{SiO}_{2}$ was deposited onto the substrates to electrically isolate the gate and drain materials (Fig S4a). $70 \mu \mathrm{m} \times 70 \mu \mathrm{m}$ square drain pads were photolithographically defined. The nanowire tips were subsequently exposed via $\mathrm{SiO}_{2}$ plasma etching (Fig. $\mathrm{S} 4 \mathrm{~b}$ ). $\mathrm{SiO}_{2}$ was removed from the top $50 \mathrm{~nm}$ of the nanowires in order to increase the contact surface area. Ni $(50 \mathrm{~nm}) / \mathrm{Pt}(30 \mathrm{~nm})$ contacts were sputtered onto the drain regions, and NiSi contacts were formed after a 5 min rapid thermal annealing treatment at $450{ }^{\circ} \mathrm{C}$ (Fig. S4c). Before the final source contact is made, photoresist is spun onto the top device side of the substrate to protect the nanowire circuitry. Al contacts were thermally evaporated onto the backside of the substrates, after oxide removal via $\mathrm{SiO}_{2}$ plasma etching and 10:1 Buffered HF. The device was subsequently annealed at $300{ }^{\circ} \mathrm{C}$, to achieve lower contact resistance.

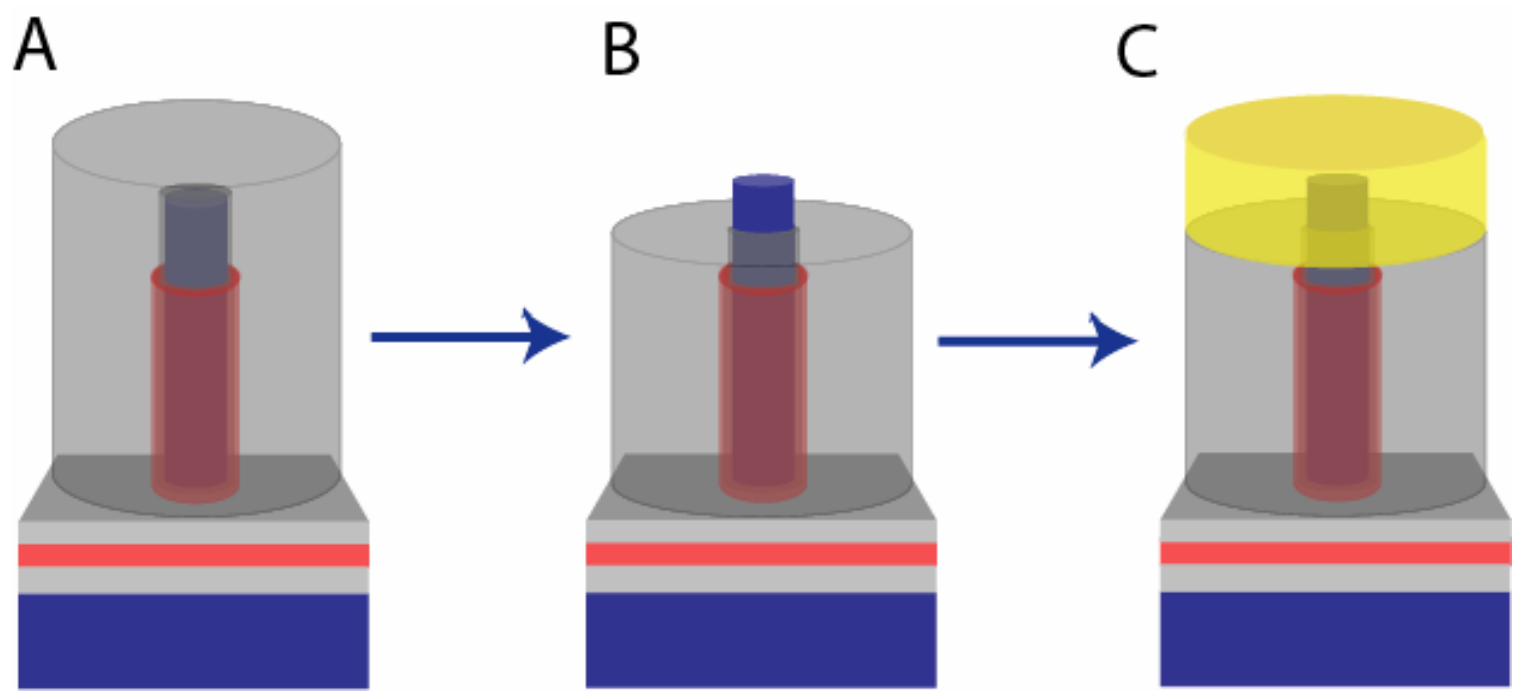

Fig. S4. Si VINFET fabrication. (A) Another layer of $\mathrm{LPCVD} \mathrm{SiO}_{2}$ is deposited onto the nanowire. (B) The nanowire tips are exposed via plasma etching of the $\mathrm{SiO}_{2}$ dielectric. (C) Ni / Pt contacts are sputtered onto the sample to form the drain electrode. $\mathrm{Cr}$ gate material is then sputtered onto the nanowires to achieve a conformal coating. Blue corresponds to the Si substrate and the Si nanowire channel, grey corresponds to the 
$\mathrm{SiO}_{2}$ dielectric material, red corresponds to the $\mathrm{Cr}$ gate metal, and yellow corresponds to the Ni drain material.

3. Threshold Voltage Analysis. In the case for our system (p-type nanowires) with a $\mathrm{Cr}$ gate electrode, the threshold voltage $\left(\mathrm{V}_{\mathrm{t}}\right)$ can be approximated by the following formula;

$$
\mathrm{V}_{\mathrm{t}}=\mathrm{V}_{\mathrm{FB}}+2 \varphi_{\mathrm{s}}+\frac{\sqrt{2 \varepsilon_{\mathrm{s}} \mathrm{qN}} \mathrm{A}\left(2 \varphi_{\mathrm{s}}\right)}{\mathrm{C}}
$$

Where $\mathrm{V}_{\mathrm{FB}}$ is the flatband voltage, $\mathrm{N}_{\mathrm{A}}$ is the acceptor concentration in $\mathrm{Si}, \mathrm{C}$ is the oxide capacitance, $\varepsilon_{\mathrm{s}}$ is the oxide permittivity, and $\varphi_{\mathrm{s}}$ is the surface potential. Since the onset of accumulation for an metal-oxide-semiconductor system occurs when the surface potential is zero $^{3}$, the threshold voltage is equal to the flatband potential. $V_{\mathrm{FB}}$ can be deduced by the following equation;

$$
\mathrm{V}_{\mathrm{FB}}=\Phi_{\mathrm{M}}-\chi-\frac{\mathrm{E}_{\mathrm{g}}}{2}-\Phi_{\mathrm{F}}
$$

Where $\Phi_{M}$ is the gate work function, $\chi$ is the electron affinity of $\mathrm{Si}$, and $\mathrm{E}_{\mathrm{g}}$ is the band gap of silicon. $\Phi_{\mathrm{F}}$ is given by the formula;

$$
\Phi_{\mathrm{F}}=\frac{\mathrm{kT}}{\mathrm{q}} \ln \left(\frac{\mathrm{N}_{\mathrm{A}}}{\mathrm{n}_{\mathrm{i}}}\right)
$$

Where $n_{i}$ is the intrinsic carrier concentration in Si. Solving equation (1) for $\mathrm{N}_{A}$ using the average observed threshold voltages gives an average carrier concentration of $3 \times 10^{16}$ $\mathrm{cm}^{-3}$. More accurate analyses of the influence of carrier concentration on threshold voltage at these small length scales can be derived using drift-diffusion simulations.

\section{4. $6.5 \mathrm{~nm}$ body VINFET}



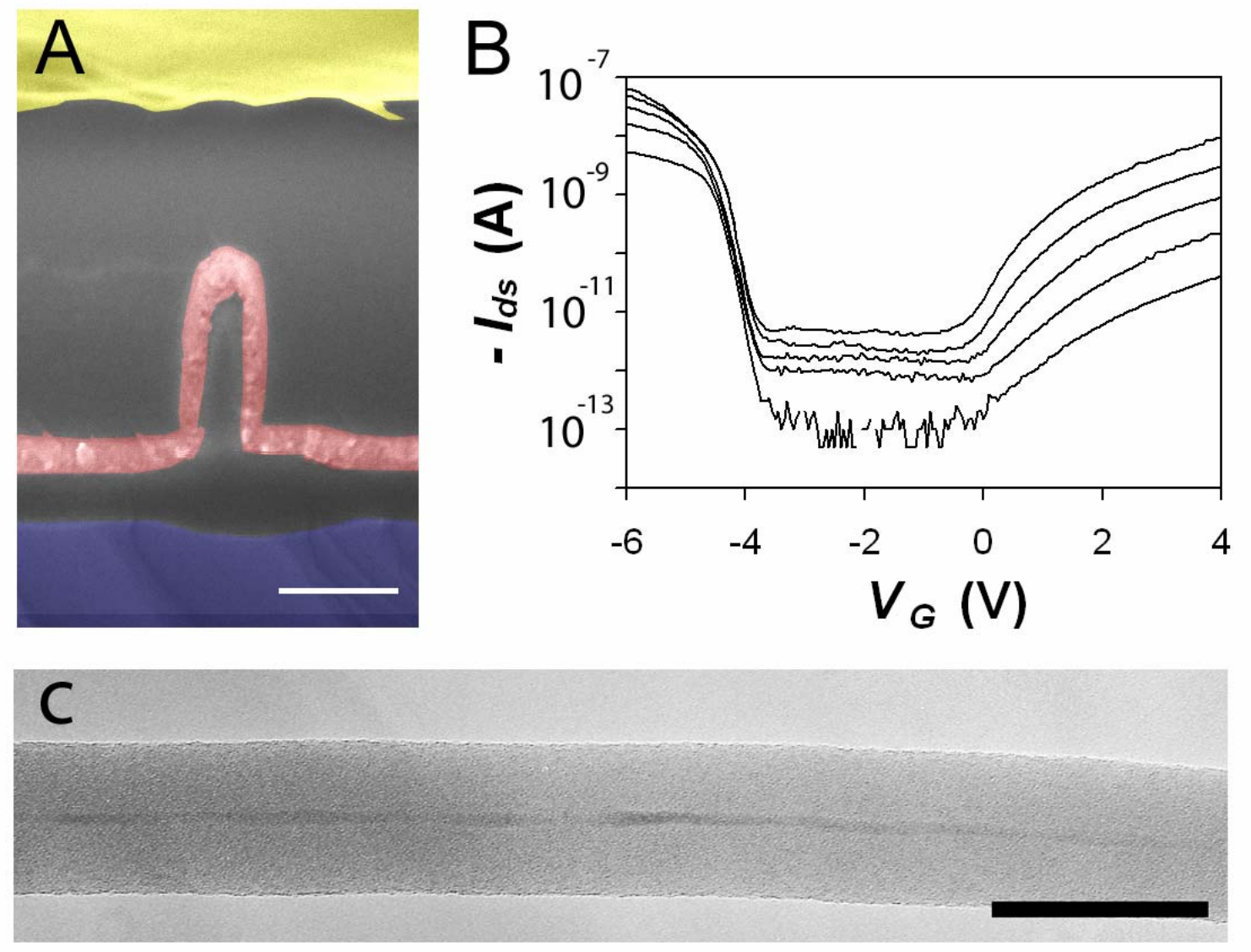

Fig. S5. Ultra-thin body VINFET. (A) Cross-sectional SEM image of a VINFET device. The scale bar corresponds to $200 \mathrm{~nm}$. False color is applied for clarity. Blue corresponds to the $\mathrm{Si}$ source, grey corresponds to $\mathrm{SiO}_{2}$ dielectric, red corresponds to the gate material, and yellow corresponds to the drain metal. The Silicon nanowire is not colored, due to the inability of resolving this feature via SEM. The Cr coverage on the front of the wire was likely stripped during cleavage. (B) $I_{d s}$ vs. $V_{g s}$ with $V_{d s}$ ranging from $-1.0 \mathrm{~V}$ to $-0.2 \mathrm{~V}$ in $0.2 \mathrm{~V}$ steps, from top to bottom, respectively, measured from a device with 48 nanowires in parallel. (C) TEM image of a $6.5 \mathrm{~nm}$ Si nanowire, obtained from the device used in (A) and (B) surrounded with a thermal oxide coating. Scale bar is $100 \mathrm{~nm}$. A typical device has a $\sim 6-7 \mathrm{~nm}$ Si nanowire diameter, surrounded by a $\sim 30$ $35 \mathrm{~nm}$ thick shell of $\mathrm{SiO}_{2}$, and a $\mathrm{Cr}$ metal gate length of $\sim 300-350 \mathrm{~nm}$. 


\section{References}

1. Huang, J.; Tao, A. R.; Connor, S.; He, R.; Yang, P. Nano Lett. ASAP, 2006.

2. Hochbaum, A. I.; Fan, R.; He, R.; Yang, P. Nano Lett. 2005, 5, 457.

3. Pierret, R. F., Semiconductor Device Fundamentals. ed.; Addison-Wesley Publishing Company, Inc.: Menlo Park, CA, 1996. 